\title{
Banana finger with giant pyogenic granuloma in lepromatous leprosy
}

\section{Mrinal Gupta}

Sudhaa Skin Centre, 35-A, Lane No-7, Tawi Vihar, Sidhra, Jammu, J\&K, India

Corresponding author: Dr. Mrinal Gupta, E-mail: drmrinalgupta@yahoo.com

Sir,

A 53-yeal old male presented to us with history of loss of sensations over the hands and feet with recurrent ulceration over the fingers for the last three years. Over a period of next two years, he also noticed multiple skin colored to erythematous nodular lesions over the trunk and the buttocks and there was gradual progression of the sensory loss with loss of distal ends of a few digits. For the last three months, the patient gave history of persistent swelling of the right middle finger and a large reddish oozing mass over the right palm, which had developed at the site of previous ulceration. There was also history of recurrent epistaxis and stuffiness of nose with congestion and watering of the eyes. On examination, there was diffuse swelling of the right middle finger with fingertip ulceration destruction of nail plate and the presence of a protuberant reddish mass over the right palm (Figs. 1 and 2). Apart from this, there was absence of distal part of little finger. The skin showed multiple skin colored to erythematous papules and nodules distributed over the trunk, extremities and the buttocks. There was complete loss of all sensations over the palmar and dorsal aspects of hands and feet and there was thickening and tenderness of multiple nerve trunks. There was pitting pedal edema and tenderness over ankle joints. Slit skin smear from the nodules revealed the presence of multiple solid stained lepra bacilli (4+). Radiological examination of extremities showed soft tissue swelling with loss of distal phalanges and destruction of middle phalanx of right middle finger (Fig. 3). All other routine hematological and biochemical investigations were normal. From the history and examination, a diagnosis of lepromatous leprosy with Type II lepra reaction with Grade II deformity with pyogenic granuloma was made and the patient was started on multi drug therapy,

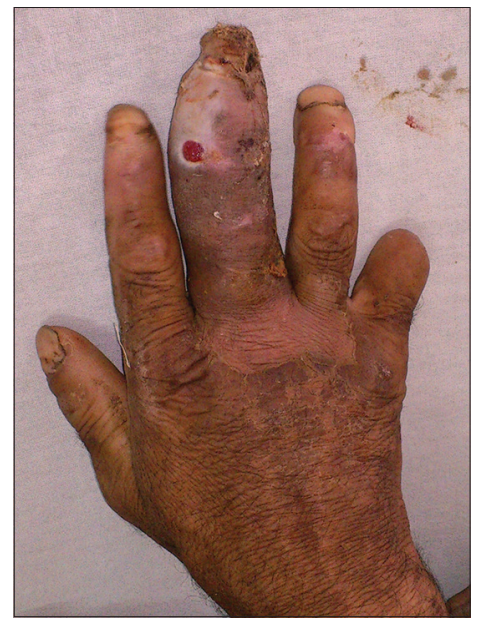

Figure 1: Sausage appearance of right middle finger with loss of distal phalanges of little finger.

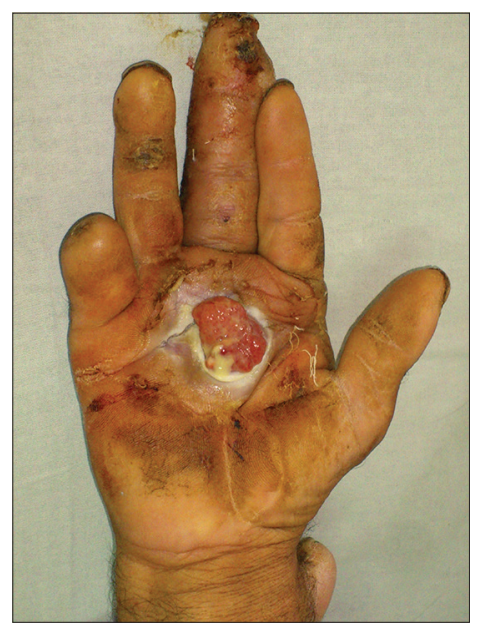

Figure 2: Pyogenic granuloma over the palm with fingertip ulceration over middle finger.

tapering doses of systemic corticosteroids and NSAIDs and the pyogenic granuloma was excised surgically. 


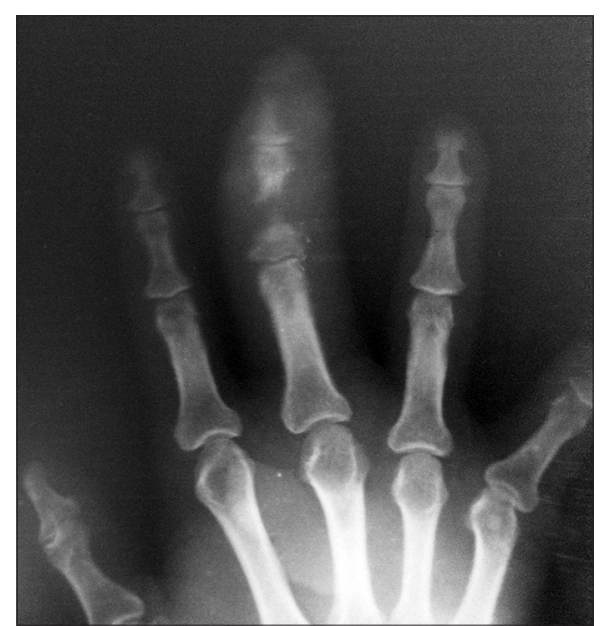

Figure 3: Radiological examination showing soft tissue swelling with destruction of middle phalanx of middle finger and loss of distal phalanges of little finger.

Leprosy, a chronic granulomatous infection caused by Mycobacterium leprae, classically manifests with cutaneous and neurological manifestations. Musculoskeletal involvement though third most common is frequently underdiagnosed and underreported. It may manifest in the form of Charcot's arthropathy, acute symmetrical polyarthritis or swollen hands and feet syndrome during lepra reactions, insidious-onset chronic symmetrical polyarthritis mimicking rheumatoid arthritis or as isolated tenosynovitis or tenosynovitis associated with arthritis or neuropathy. Banana finger (sausage appearance of digits) is a frequent clinical finding in leprous dactylitis and is commonly seen as a manifestation of Type 2 lepra reaction [1]. Delay in diagnosis and management may be detrimental and may result in deformities and loss of function.

\section{REFERENCE}

1. Pereira HL, Ribeiro SL, Sato EI. Rheumatic manifestations in leprosy. Acta Reumatol Port. 2008;33:407-14.

Copyright by Mrinal Gupta. This is an open access article distributed under the terms of the Creative Commons Attribution License, which permits unrestricted use, distribution, and reproduction in any medium, provided the original author and source are credited.

Source of Support: Nil, Conflict of Interest: None declared. 